\title{
VaR Model Measure Exchange Rate Risk Based on GARCH Approach and EVT Theory
}

\author{
Jie Yang \\ School of Economics and Management \\ Yunnan Normal University \\ Kunming, China
}

\author{
Shaozong Zhang \\ Finance Department \\ Yunnan Normal University \\ Kunming, China
}

\begin{abstract}
In recent years, financial disasters have emphasized the importance of effective risk management for financial regulators and market practitioners who have become particularly sensitive to changes in the assets value. The use of quantitative risk measures has become an essential management tool to be placed in parallel with the models of returns. In this paper, we introduce all kinds of GARCH models and EVT theory and apply those method to measure exchange rate risk of Chinese exchange market. Firstly, we examine the the heteroscedasticity of the return series of USD/RMB and EUR/RMB data, the results suggest that there is obvious heteroscedasticity. Secondly, we choose the best GARCH model to filter the return series to i.i.d residual series and employ extreme value theory to estimate the tails of those i.i.d series, we find the degree of fitting of GPD is very high. Finally, we calculate corresponding VaR estimates, which can help investor to measure exchange risk accurately.
\end{abstract}

Keywords-VaR; GARCH; EVT; Exchange rate; Market Risk Measurement

\section{INTRODUCTION}

In the past decades, financial disasters have emphasized the importance of effective risk management for financial regulators and market practitioners who have become particularly sensitive to changes in the assets value. The use of quantitative risk measures has become an essential management tool to be placed in parallel with the models of returns. These measures are used for investment decisions, supervisory decisions, risk capital allocation and external regulation. In the fast paced financial world, effective risk measures must be as responsive to news as are other forecasts and must be easy to grasp even in complex situations. Generally speaking, risk management consists of four complementary steps: identification, assessment, monitoring and control (mitigation). Among them, risk assessment (measurement) is an important prerequisite for the following risk control. The method of risk measurement has undergone many changes from the traditional tool -the standard deviation to the slightly more complicated sensitive indicators of risk factors (e.g., duration and Gamma) to the now widely used but highly controversial method of Valueat-Risk (VaR).

Since Value-at-Risk received its first wide representation in July 1993 in the Group of Thirty report, the number of uses of-and uses for-Value-at-Risk have increased dramatically. But it is important to recognize that the Valueat-Risk technique has gone through significant refinement and passed essential process changes since it originally appeared. Theoretical research that relied on the Value-atRisk as a risk measurement was initiated by Jorion(1997) and Dowd(1998), who applied the Value-at-Risk approach based on risk management emerging as the industry standard by choice or by regulation. Value-at-Risk based management by financial as well as non-financial institutions was researched and described by J.P. Morgan(2000). Its wide use occurs form the fact that Value-at-Risk is an easily interpretable summary measure of risk and also has an attractive explanation, as it allows its users to focus attention on the normal market condition in their routine operations. Value-at-Risk models aggregate the several components of price risk into a single quantitative measure of the potential for losses over a specified time horizon.

Recognition of such models by financial and regulatory communities is evidence of their growing use. For instance, financial regulation, like the Basel Committee on Banking Supervision, requires U.S. banks to use VaR to determine the minimum capital to support their trading portfolios. Since 1998 the Securities and Exchange Commission requires registrants to provide quantitative information about market risk with VaR being one of the disclosure alternatives.

The rest of paper is structured as follows: in section II, a short overview of general concept of $\mathrm{VaR}$ and ES will be presented. In section III, we review the ARCH type model including GARCH models. Meantime, an overview about extreme value theory (EVT) will also be presented. In section IV, we focus on an application of VaR model based on GARCH approach and EVT on Chinese Exchange rate including USD/RMB and EUR/RMB data. In section V, we shall give a summary of the full text.

\section{VAR MODELS AND EXTREME VALUE THEORY}

\section{A. General Concept of VaR and ES}

In this section, we firstly give definitions of $\mathrm{VaR}$ and ES and discuss their use and basic properties. Let $X$ be a random variable with the cumulative distribution function 
$F_{X}=P\{X \leq x\} . X$ may represent the loss or gain of a return or portfolio. In this paper, $X$ has the meaning of loss.

Definition 1 (Value-at-Risk) The VaR of $X$ with confidence level $\alpha \in(0,1)$ is

$$
\operatorname{VaR}_{\alpha}(X)=\inf \left\{x \in R, F_{X}(x) \geq \alpha\right\}
$$

By above definition, $\operatorname{VaR}_{\alpha}(X)$ is a left $\alpha$ quantile of the random variable $X$. For a given time horizon $\mathrm{t}$ and confidence level $\alpha$, the VaR of a portfolio is the potential loss in the portfolio's market value over the time horizon $t$ that is exceeded with probability $1-\alpha$. VaR has become a popular risk measure for risk management both for the purposes of reporting and measurement of capital adequacy. Despite its wide acceptance, it is not a coherent risk measure. A coherent risk measure should satisfy the axioms of translation invariance, subaddivity, positive homogeneity, and monotonicity. Unfortunately, VaR lacks sub-additivity.

Another informative measure of risk is the Expected Shortfall (ES), which is also known as Mean Excess Loss or Tail VaR. ES is a more consistent measure of risk since it is subaddivity and convex. Next, we give a formal definition of ES.

Definition 2 (Expected Shortfall) For random variable $X$ with conditional distribution function, ES is the conditional expectation of the loss above $\mathrm{VaR}$ for the time horizon $\mathrm{t}$ and the confidence level $\alpha$, i.e.

$$
E S_{\alpha}(X)=E[X \mid X>V a R]=\int_{-\infty}^{+\infty} z d F_{X}^{\alpha}(z)
$$

Where

$$
F_{X}^{\alpha}(z)=\left\{\begin{array}{cl}
0 & , \text { when } z<\operatorname{VaR}_{\alpha}(X) \\
\frac{F_{X}(z)-\alpha}{1-\alpha} & , \text { when } z \geq \operatorname{VaR}_{\alpha}(X)
\end{array}\right.
$$

\section{B. Extreme Value Theory and POT Method}

Extreme Value Theory (EVT) is a classical topic in probability theory. Many books and surveys are available on the subject, here we intend to give some intuition and basic results of EVT.

Generally there are two related ways of identifying extremes in real data. Let $X_{i}(i=1,2, \ldots)$ denote a random variable which may represent daily losses or returns. The first approach considers the maximum (or minimum) the variable takes in successive period, for instance weeks, months or years. These selected observations constitute the extreme events, a method that determines these selected extreme values, is called block maxima method (BMM); the second approach focuses on the realizations which exceed a given threshold, all exceed the threshold $u$ constitute extreme events. The BMM is the traditional method used to analyze data with seasonality. However, the Peak Over Threshold (POT) method used data more efficiently and seems to be a popular choice method in recent applications. In following part, the POT method is introduced in detail.

Let $X_{i}(i=1,2, \ldots)$ denote a sequence of i.i.d random variables with an unknown distribution function $F(x)$. We focus on the estimating the distribution function $F_{u}$ of values of $x$ above a certain threshold $u$. The distribution function $F_{u}$ is called the conditional excess distribution function(cedf). Formally

$$
F_{u}(y)=P\{X-u \leq y \mid X>u\}, y \geq 0
$$

Where $X$ is a random variable, $u$ is a given threshold. Based on conditional probability formula, we have

$$
F_{u}(y)=P\{X-u \leq y \mid X>u\}=\frac{F(u+y)-F(u)}{1-F(u)}=\frac{F(x)-F(u)}{1-F(u)}
$$

Define also the Generalized Pareto Distribution (GPD) as:

$$
G_{\xi, \beta}(y)= \begin{cases}1-\left(1+\xi \frac{y}{\beta}\right)^{-\frac{1}{\xi}} & \text { if } \xi \neq 0 \\ 1-e^{-\frac{y}{\beta}} & \text { if } \xi=0\end{cases}
$$

The following is a crucial property of GPD, due to Pickands (1975). For a large class of underlying distribution function $F$, the conditional excess distribution function $F_{u}$, for $u$ enough large, is satisfied as $\lim _{u \rightarrow \infty} \sup _{0<x<x_{F}-u}\left|F_{u}(x)-G_{\xi, \beta}(x)\right|=0$. Where $x_{F}$ is the right endpoint (which could be infinite) of the distribution $F$. This result indicates that GPD becomes an accurate approximation of the excess distribution function $F_{u}$.

The parameters of the GPD can be estimated by maximizing the likelihood function, once the threshold has been chosen. The log-likelihood based on (5) computed on the $\mathrm{n}$ upper order statistics is:

$$
L(\xi, \beta \mid y)= \begin{cases}-n \log \beta-\left(\frac{1}{\xi}+1\right) \sum_{i=1}^{n} \log \left(1+\frac{\xi}{\beta} y_{i}\right) & \text { if } \xi \neq 0 \\ -n \log \beta-\frac{1}{\xi} \sum_{i=1}^{n} y_{i} & \text { if } \xi=0\end{cases}
$$

An attractive feature of GPD is that we have a close form formula for $\mathrm{VaR}$. in fact, from (4) we have

$$
F(x)=[1-F(u)] F_{u}(y)+F(u)
$$

after replacing $F_{u}$ by the GPD and $F(u)$ by its estimate $\left(n-N_{u}\right) / n$, where $n_{\text {is }}$ the total number of observation and $N_{u}$ is the number of observations above the threshold $u$,we can get the following result

$$
F(x)=1-\frac{N_{u}}{n}\left(1+\xi \frac{x-u}{\beta}\right)^{-\frac{1}{\xi}}
$$


Form (6), the calculating $\mathrm{VaR}$ of confidence level ${ }^{q}$ is a quite easy task. We only need solve equation $F(\operatorname{VaR})=q$. We then have

$$
\operatorname{VaR}_{q}=u+\frac{\beta}{\xi}\left[\left(\frac{n}{N_{u}}(1-q)\right)^{-\xi}-1\right]
$$

Similarly, we can get a close formula for $E S_{q}$

$$
E S_{q}=V a R_{q}+\frac{\beta+\left(V a R_{q}-u\right) \xi}{1-\xi}=\frac{V a R_{q}}{1-\xi}+\frac{\beta-\xi u}{1-\xi}
$$

\section{ARCH AND GARCH MODELS}

\section{A. GARCH Models}

Since the introduction of ARCH model introduced by Engel, GARCH models, introduced by Bollerslev, generalized Engle's earlier ARCH models to include autoregressive (AR) as well as moving average (MA) terms. GARCH models can be more parsimonious (use fewer parameters), increasing computational efficiency. After that, there has been a numerous extension of GARCH models emerged to specify the conditional variance models. The class of GARCH models has become extremely popular for application to financial data over past 20 years. In follow part, the GARCH class of models is explained as follow.

1) $\operatorname{GARCH}(p, q)$

A typical GARCH(p,q) process composed of two parts. The first part is the mean function

$$
y_{t}=c+\sum_{i=1}^{R} \phi_{i} y_{t-i}+\varepsilon_{t}+\sum_{j=1}^{M} \theta_{j} \varepsilon_{t-j}+\sum_{k=1}^{N_{x}} \beta_{k} X(t, k)
$$

The above equation implies that $y_{t}$ follows a $\operatorname{ARMA}(R, M)$ process. The second part is composed of the variance equation. The GARCH model can be characterized by the assumption that the conditional heteroskedastic series satisfies

$$
\left\{\begin{array}{l}
\varepsilon_{t}=\sigma_{t} \eta_{t}, \eta_{t} \sim \text { i.i.d. } N(0,1) \\
\sigma_{t}^{2}=k+\sum_{i=1}^{p} G_{i} \sigma_{t-i}^{2}+\sum_{j=1}^{q} A_{j} \varepsilon_{t-j}^{2}
\end{array}\right.
$$

where

$p \geq 0, q>0, k>0, G_{i}>0(i=1,2, . ., p), A_{j}>0(j=1,2, . ., q)$, and $\sum_{i=1}^{p} G_{i}+\sum_{j=1}^{q} A_{j}<1$

\section{2) $\operatorname{EGARCH}(p, q)$}

Exponential GARCH model is proposed by Nelson in 1991[4]. The variance equation of $\operatorname{EGARCH}(p, q)$ is the

$$
\log \sigma_{t}^{2}=k+\sum_{i=1}^{p} G_{i} \log \sigma_{t-i}^{2}+\sum_{j=1}^{q} A_{j}\left[\frac{\left|\varepsilon_{t-j}\right|}{\sigma_{t-j}}-E\left\{\frac{\left|\varepsilon_{t-j}\right|}{\sigma_{t-j}}\right\}\right]+\sum_{j=1}^{q} L_{j}\left(\frac{\varepsilon_{t-j}}{\sigma_{t-j}}\right)
$$

(11)

Since EGARCH model take a log transformation, the conditional heteroskedasticity is guaranteed to be positive. No restrictions need to be imposed on coefficients.
Furthermore, the conditional heteroskedasticity depends on the absolute magnitude of shocks and reflects the effect of the shock direction. If the parameter estimate is $L_{j} \neq 0$, it suggests that the existence of the asymmetry. If $L_{j}<0$ is result, bad news $\left(\varepsilon_{t}<0\right)$ cause more volatility than good news $\left(\varepsilon_{t}>0\right)$. On the contrary, it suggests good news cause more volatility than bad news.

\section{3) $\operatorname{GARCH}-G J R(p, q)(T G A R C H)$}

In addition, a model reflecting of financial market asymmetric volatility is called threshold GARCH(TGARCH) introduced by Glosten, Jagannathan and Runkel in 1993. Its conditional heteroskedasticity function is

$$
\sigma_{t}^{2}=k+\sum_{i=1}^{p} G_{i} \sigma_{t-i}^{2}+\sum_{j=1}^{q} A_{j} \varepsilon_{t-j}^{2}+\sum_{j=1}^{q} L_{j} \lambda_{t-j} \varepsilon_{t-j}^{2}
$$

where $\sum_{i=1}^{p} G_{i}+\sum_{j=1}^{q} A_{j}+\frac{1}{2} \sum_{j=1}^{q} L_{j}<1$, and $\lambda_{t-j}= \begin{cases}1, & \varepsilon_{t-j}<0 \\ 0, & \varepsilon_{t-j} \geq 0\end{cases}$

$L_{j}$ is called as the leverage, if $L_{j}>0$, it means that the effect of bad news $\left({ }^{\varepsilon_{t-j}}<0\right)$ is larger than good news.

\section{EMPIRICAL STUDY}

In this section, we will apply those theories to make empirical research. Firstly, we choose Chinese foreign exchange rate as samples of our empirical study. Secondly, we investigate the statistical features and fat-tail behavior of samples. Thirdly, we attend to find out a good candidate of the $\log$ return distribution and build a fitted VaR model based on it.

\section{A. Sample Selection and Data Statistical Features}

China's official exchange rates mainly involve five currencies: U.S. dollar(USD), Euro(EUR), Japanese Yen(JPY), Hong Kong dollar(HKD) and British Pound Sterling(GPD). In this paper, we will use the exchange rate of USD/RMB and EUR/RMB as our main object. All the data come from the middle price of daily exchange rate of RMB published on the official website of State Administration of Exchange (http://www.safe.gov.cn/model_safe/index.html). The data that we use in this paper ran from July 25, 2005 to March 31, 2017, altogether 2845 data. The reason for choosing this period is that we hope our study can reveal the main feature of the Chinese exchange market after the Chinese currency reform. It is known fact the major change in Chinese foreign exchange market started at July 21, 2005 when China began to carry out a managed floating exchange rate policy which is based on the market supply and demand and makes adjustments in accordance with the reference currency basket. The Figure 1 below shows the trend of the price sequence of USD and EUR in the chosen period "Fig. 1". 


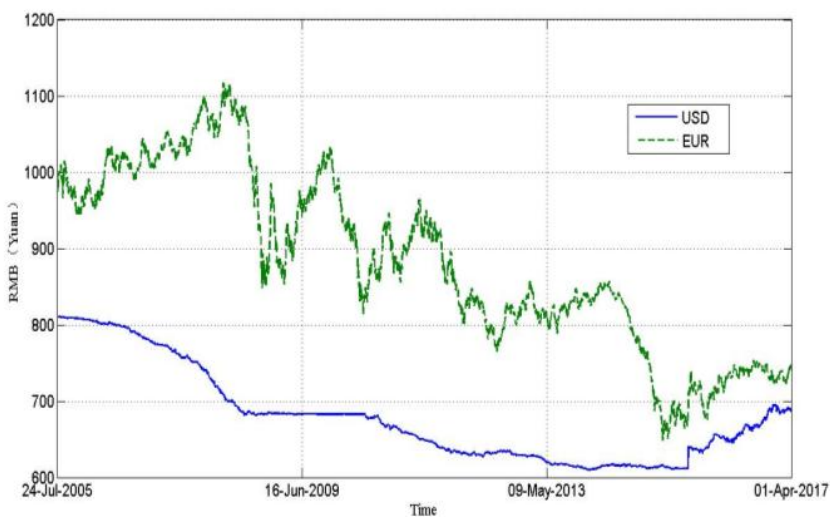

Fig. 1. USD and EUR exchange rate time series.

We introduce our notation now. We will use $r$ to denote the $\log$-return, i.e., ${ }_{i}=100\left[\log \left(P_{t}\right)-\log \left(P_{t-1}\right)\right]$, where $P_{t}$ is the closing quotation price of the daily exchange rate. Using mathematical software, such as Matlab2016(b), we could easily get the statistical features of these two types of foreign exchange data, and the results are recorded in below: "Table I" Statistical features of log-return.

TABLE I. STATISTICAL FEATURES OF LOG-RETURN

\begin{tabular}{|c|c|c|c|c|c|c|}
\hline & Mean & Std & Skewness & Kurtosis & $\begin{array}{c}\mathbf{J}-\mathbf{B} \\
\text { statistic }\end{array}$ & Prob \\
\hline USD & $-\overline{0.00056}$ & 0.0012 & 2.5643 & 42.3371 & 18648 & 0.0000 \\
\hline EUR & $\begin{array}{l}- \\
0.00088\end{array}$ & 0.0062 & -0.1741 & 10.0108 & 5838.8 & 0.0000 \\
\hline
\end{tabular}

From "Table I" and "Fig. 2", we find some typical statistical features of financial time series, such as leptokurtosis, fat tails and non-normal distribution. All these significant features should be taken into consideration when we choose $\mathrm{VaR}$ models to estimate exchange rate risks.
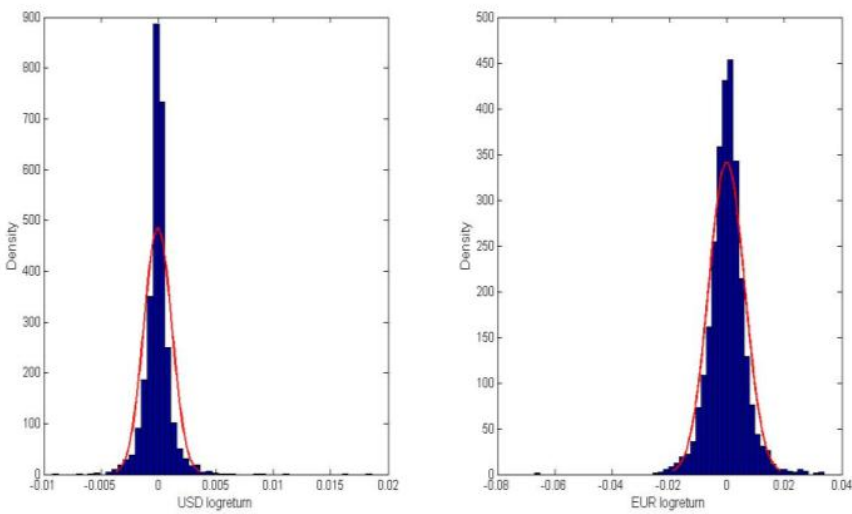

Fig. 2. USD and EUR exchange rate log-returns density and Normal density.

\section{B. GARCH Model Selection about Logreturn}

Return series are generally required stationary to build a model. So we conduct ADF (unit root) test, the result showed that the logreturn series of USD and EUR are stationary. In addition, we also conduct Engle's ARCH test for heteroscedasticity on the residual, the results are in "Table II", which shows that there is obvious heteroscedasticity. It is reasonable to build a GARCH model to fit the log-return series.

TABLE II. TEST FOR HETEROSCEDASTICITY

\begin{tabular}{|c|l|l|l|l|}
\hline $\begin{array}{c}\text { orde } \\
\mathbf{r}\end{array}$ & $\begin{array}{c}\text { LM-statistics } \\
\text { For USD }\end{array}$ & pValue & $\begin{array}{c}\text { LM- statistics } \\
\text { For EUR }\end{array}$ & pValue \\
\hline $\mathbf{1}$ & 55.7321 & 0.000376 & 231.7028 & 0 \\
\hline $\mathbf{2}$ & 71.6247 & 0.001232 & 235.4935 & 0 \\
\hline $\mathbf{3}$ & 110.3689 & 0.002645 & 235.9931 & 0 \\
\hline
\end{tabular}
characteristics, we assess the performance of the GARCH$\mathrm{t}(1,1)$, EGARCH-t $(1,1)$ and GARCH-t-GJR(1,1) model according to AIC-BIC criterion, where $t$ means their marginal distributions both obey general Student $t$ distribution. The estimated results are shown in "Table III" and "Table IV". For USD data, we find that the AIC and BIC of EGARCH-t $(1,1)$ is the smallest, which indicates that EGARCH-t $(1,1)$ model is the best to fit logreturn's volatility. But for EUR data, the GARCH-t $(1,1)$ model is probably the best one. The best estimation is in formula (13) and (14).

TABLE III. AIC-BIC CRITERION FOR USD GARCH MODELS

\begin{tabular}{|l|l|l|l|}
\hline & \multicolumn{1}{|c|}{ GARCH-t $(\mathbf{1 , 1})$} & EGARCH-t(1,1) & $\begin{array}{c}\text { GARCH-t- } \\
\text { GJR(1,1) }\end{array}$ \\
\hline $\boldsymbol{A I C}$ & -13443 & -13812 & -13446 \\
\hline $\boldsymbol{B I C}$ & -13418 & -13781 & -13416 \\
\hline
\end{tabular}

TABLE IV. AIC-BIC CRITERION FOR EUR GARCH MODELS

\begin{tabular}{|l|l|l|l|}
\hline & \multicolumn{1}{|c|}{ GARCH-t $(\mathbf{1 , 1})$} & EGARCH-t(1,1) & \multicolumn{1}{c|}{$\begin{array}{c}\text { GARCH-t- } \\
\text { GJR(1,1) }\end{array}$} \\
\hline AIC & -8367.9 & -8358.5 & -8367.7 \\
\hline BIC & -8342.8 & -8328.4 & -8337.6 \\
\hline
\end{tabular}

$r_{U S D_{t}}=-8.1391 \times 10^{-6}+\varepsilon_{t}$

$(-2.4371)$

$\log \sigma_{t}^{2}=-0.0141+1 \times \log \sigma_{t-1}^{2}+0.2066 \frac{\left|\varepsilon_{t-1}\right|}{\sigma_{t-1}}-0.03915 \frac{\varepsilon_{t-1}}{\sigma_{t-1}}$

$$
\begin{array}{llll}
(-0.31) & (344.5) \quad(6.0413) \quad(-1.5986)
\end{array}
$$

$$
\left\{\begin{aligned}
r_{E U R_{t}}= & 9.1636 \times 10^{-5}+\varepsilon_{t} \\
& (0.6245) \\
\sigma_{t}^{2}= & 2 \times 10^{-7}+0.958 \sigma_{t-1}^{2}+0.03547 \varepsilon_{t-1}^{2} \\
(1.1899) & (-94.04)
\end{aligned}\right.
$$

\section{VaR Calculation}

From GARCH models discussed above, we attained the series of standardized residuals of the log-return. By employing EVT, we can estimate parameters by using maximizing the likelihood function (7). Estimated results are shown in "Table V". "Fig. 3" shows the effect of the residual data fitted by GPD and the corresponding Quantile-Quantile plot (Q-Q plot), both suggest very satisfactory fitting has been achieved. 

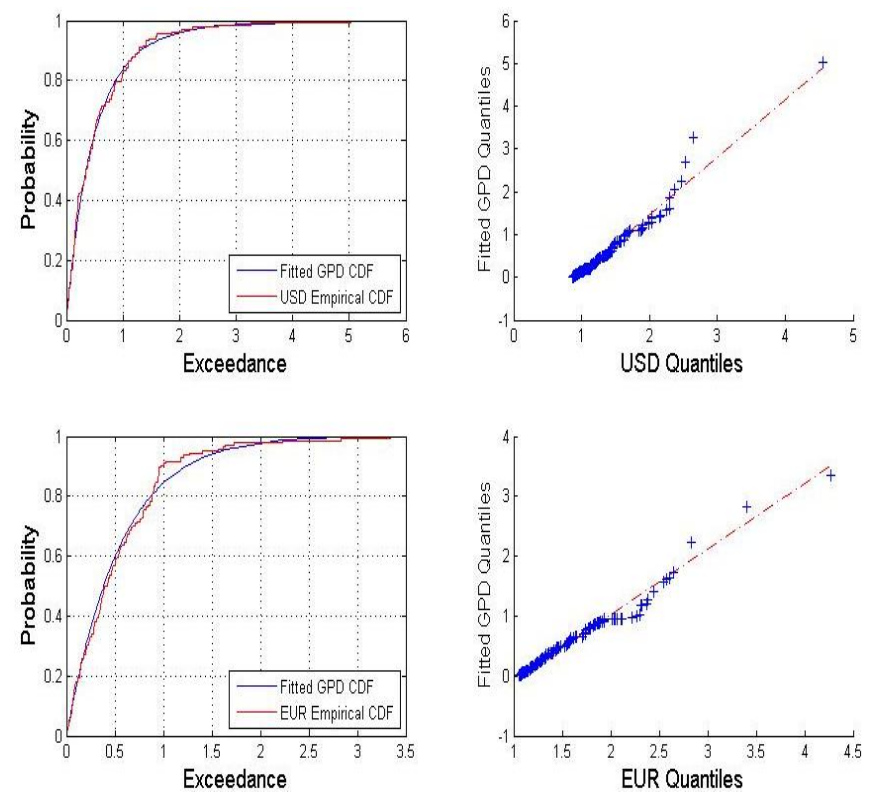

Fig. 3. GPD fitted to the USD and EUR residuals (right tail).

TABLE V. ESTIMATED PARAMETERS OF GPD

\begin{tabular}{|c|c|c|c|c|c|c|c|}
\hline & \multicolumn{7}{|c|}{ Right Tail } \\
\hline & $\boldsymbol{u}$ & $\begin{array}{l}\text { Exce } \\
\boldsymbol{e} \\
\text { dance }\end{array}$ & ratio & $\boldsymbol{s}$ & $\operatorname{se}(\xi)$ & $\hat{\beta}$ & $\operatorname{se}(\hat{\beta})$ \\
\hline $\boldsymbol{U S D} \boldsymbol{D}$ & $\begin{array}{l}0.859 \\
2\end{array}$ & 263 & $\begin{array}{l}11.97 \\
\%\end{array}$ & $\begin{array}{l}0.158 \\
5\end{array}$ & 0.100 & $\begin{array}{l}0.474 \\
4\end{array}$ & 0.0625 \\
\hline $\begin{array}{c}\boldsymbol{E} \boldsymbol{U} \\
\boldsymbol{R}\end{array}$ & $\begin{array}{l}1.317 \\
9\end{array}$ & 190 & $8.64 \%$ & $\begin{array}{l}0.016 \\
1\end{array}$ & $\begin{array}{l}0.090 \\
3\end{array}$ & $\begin{array}{l}0.481 \\
4\end{array}$ & 0.0656 \\
\hline & \multicolumn{7}{|c|}{ Left Tail } \\
\hline & $\boldsymbol{u}$ & $\begin{array}{l}\text { Exce } \\
\boldsymbol{e} \\
\text { dance }\end{array}$ & ratio & $\xi$ & $\operatorname{se}(\xi)$ & $\hat{\beta}$ & $\operatorname{se}(\hat{\beta})$ \\
\hline $\boldsymbol{U S D}$ & -1.344 & 219 & $9.99 \%$ & $\begin{array}{l}0.076 \\
6\end{array}$ & $\begin{array}{l}0.092 \\
8\end{array}$ & $\begin{array}{l}0.543 \\
3\end{array}$ & 0.0720 \\
\hline $\begin{array}{c}\boldsymbol{E U} \\
\boldsymbol{R}\end{array}$ & -1.292 & 219 & $9.99 \%$ & $\begin{array}{l}0.229 \\
0\end{array}$ & $\begin{array}{l}0.102 \\
4\end{array}$ & $\begin{array}{l}0.394 \\
4\end{array}$ & 0.0544 \\
\hline
\end{tabular}

Furthermore, we applies the conditional variance function to estimate volatility ${ }^{\sigma_{t+1}}$, and employed the VaR of the sequence of residuals to conditional mean function $r_{t+1}=c+\sigma_{t+1} Z_{t+1}$, where $Z_{t+1}$ is standardized residual. Then, the $\mathrm{VaR}$ estimates for returns of exchange rate can be obtained. Table VI displays the VaR of right tail and left tail of the USD and EUR with high (99\% or 1\%) and low (95\% or $5 \%$ ) confidence level.

TABLE VI. VAR ESTIMATES OF EMPIRICAL DATA

\begin{tabular}{|l|c|c|c|c|}
\hline & \multicolumn{2}{|c|}{ Right Tail } & \multicolumn{2}{c|}{ Left Tail } \\
\hline & VaR $_{0.99}$ & VaR $_{0.95}$ & $V R_{0.01}$ & $V_{0.05}$ \\
\hline $\boldsymbol{U S D}$ & 0.030 & 0.021 & -0.0213 & -0.0184 \\
\hline EUR & 0.023 & 0.017 & -0.0115 & -0.0057 \\
\hline
\end{tabular}

\section{CONCLUSION}

The research of risk measurement is the ubiquitous question in the field of finance and economics. Unlike traditional method that directly use Extreme Value Theory to the raw empirical data, in this paper we apply ARMA-
GARCH models to EVT to capture the statistical features of return series of Chinese exchange market. First of all, we choose the best GARCH model to filter return series to independent identical distribution's residual series and select t distribution as the marginal distribution to compensate for the fat tails. Then we employ the GPD to analyze the standard residual series and estimate relevant parameters used by POT method. Finally we examine the Chinese exchange rate data USD/RMB and EUR/RMB, and calculate corresponding VaR estimates, which may provide investor with more accurate measurement of foreign exchange risk.

\section{REFERENCES}

[1] Javier F. Navas, "Calculation of Volatility in a Jump-Diffusion Model,” The Journal of Derivatives, Oct, 2003, pp1-17

[2] Engle R, "Autoregressive Conditional Heteroskedasticity with Estimates of The Variance of United Kingdom Inflation," Journal of Finance, 1982, of United 50 (3), pp.812-851

[3] Bollerslev T., "Generalized Autoregressive Conditional Heteroskdasticity,” Journal of Econometrics, 1986, 31, pp. 307-327.

[4] Nelson, "Conditional Heteroskdasticity in Asset Returns: A New Approach,” Econometrics, 1991, pp. 347-370.

[5] Gencay. R, and Selcuk. F, "Extreme Value Theory and Value-atRisk:Relative Performance in Emerging Markets," International Journal of Forecasting, 2004, 20.

[6] John C. Hull, "Risk Management and Financial Institutions," China Machine Press, 2008, pp. 144-149.

[7] PAN Zhibin. "Risk measurement in financial market". Shanghai: Shanghai Academy of Social Sciences Press, China, 2008, pp.78-115

[8] WANG Xinyu. "Measure Methods and Empirical Analysis of Financial Market Risk," Beijing: Economic Management Press, China, 2008, pp. 34-85. 